\title{
Health care professionals' compliance to tracheal suctioning policy at a tertiary care hospital
}

\begin{abstract}
Objective: To assess the compliance of tracheal suctioning practices against the standard guidelines of a tertiary care hospital, with regard to the participants' professional characteristics.

Methods: Using cross sectional study design, forty health care professionals (HCPs) were assessed twice, using a quantitative structured observational design, for tracheal suctioning practices, in one of the surgical care units of the tertiary care hospital. Tracheal suctioning policy which was used as a tool, its inter-rater reliability was tested and each step showed the Kappa value of 0.65 to 1.000. Data was analyzed using epidata info version 3.5 .1 and SPSS version 19.

Results: From a total of 80 observations, 30\% showed compliance, $50 \%$ partial compliance whereas, $20 \%$ indicated non-compliance.

Conclusion: The study results highlight the need for improving the overall compliance with the tracheal suctioning guidelines of evidence based practices to minimize the adverse effects and enhance patient safety related to tracheal suctioning practices. The current study also provides an opportunity to motivate the HCPs compliant in the practices of tracheal suctioning by appreciate their competence.
\end{abstract}

Keywords: tracheal suctioning, quality care, evidence based practice, health care facility
Volume 7 Issue 3 - 2020

\author{
Rozina Khimani, Fauziya Ali, Salma Rattani, \\ and Sohail Awan \\ Aga Khan University, Pakistan
}

Correspondence: Rozina Khimani, Aga Khan University, Pakistan, Email rozina.khimani@aku.edu

Received: July 25, 2020 | Published: September 0I, 2020

\section{Introduction}

Tracheal suctioning is one of the critical interventions which facilitate effortless breathing patterns through effective secretion management. However; it is associated with mild injury to the life threatening arrhythmias and, sometimes with cardiac arrest. Therefore, it requires competence to carry out this intervention in order to prevent complications in patients. Many studies have been done on actual suctioning practices, and on suggested guidelines and protocols for safe suctioning skills. If the practices of suctioning are not safe or not proven to be evidence based practices, this can put patients in life threatening complications. Therefore, the suctioning practices of health care professionals are crucial for minimizing the risk of complications associated with suctioning skills.

\section{Methods}

Descriptive cross sectional non participant structured observational study design was used to observe the compliance of tracheal suctioning practices among health care professionals with respect to standard protocol used at a tertiary care hospital (TCH) in Karachi, Pakistan. Purposive sampling method was used to select a surgical unit; whereas a universal sampling technique was used to select study participants. All health care professionals working in surgical unit including nurses, critical care technicians (CCTs), and physiotherapists were included in the study. Data was collected at a single point of time and was analyzed in descriptive and inferential statistics. This study was reviewed and approved by the ethics committee of the selected setting.

\section{Results}

\section{Demographic profile of the participants}

A total of 40 participants were enrolled in the study. These included $67.5 \%$ nurses, $20 \%$ critical care technicians (CCTs) and $12.5 \%$ physiotherapists. Among all $30 \%$ were female and $70 \%$ were male. Their experience ranged from 1- >10 years however, majority $(47.5 \%)$ had 4- 7 years of experience.

\section{Number of shifts covered}

Each participant's $(n=40)$ tracheal suctioning practices were observed twice. A total of 80 observations were collected in three shifts (morning, evening, and night), out of which $50 \%$ of the observations were carried out in the morning shift, $36 \%$ in the evening shift, and $14 \%$ of the observations were carried out in the night shift. Since the observations of the evening and night shifts were lesser than the observations carried out in the morning, therefore, observations covered in the evening and night shifts were merged, in order to get the inferential analysis, and were then compared with the morning shift. Compliance of lesser then and equal to $50 \%$ on the tool was considered to be non-compliant; performance scores from 51\% -60\% were considered partially compliant; performance scores between $61 \%-80 \%$ were considered compliant; and, a score above $80 \%$ was considered good compliance. Among all $(n=40) 30 \%$ were compliant, $50 \%$ were partial compliant and $20 \%$ of the observations fell in the category of non-compliance. Moreover, pair t test was run to assess the difference of the two observations of each participant and the $\mathrm{P}$ 
value was calculated. The findings revealed that the P-value of 0.67 indicated no significant differences within the two sets of observations of each participant.

\section{Level of compliance with regard to the phases of tracheal suctioning}

Observation of tracheal suctioning was divided into three phases; pre-suctioning, suctioning, and post suctioning phase including documentation. Majority of the observations $(62.5 \%)$ showed noncompliance in the pre-suctioning phase. However, practices in the suctioning phase showed a $65 \%$ level of compliance. The observations of the post-suctioning phase showed the level of compliance to be $92.5 \%$, while the documentation phase showed only a $7.5 \%$ level of compliance. Observation gathered to assess the compliances with regard to the individual components of the suctioning phase is presented through Table 1.

\section{Difference of compliance among HCPs}

This section presents the differences in the level of compliance among HCPs, with regard to their professional characteristics, through the Fisher Exact test $\mathrm{x}^{2}$. The levels of compliance among nurses were $25.9 \%$, as compared to CCTs and physiotherapists, which were 25 and $60 \%$, respectively. However, $48.2 \%$ of the observations of the nurse's fell under the category of partially compliant as compared to the CCTs and physiotherapists, which showed $62.5 \%$ and $40 \%$ compliance respectively. Moreover, $25.9 \%$ of the observations of the nurses showed non-compliance with the tracheal suctioning practices, and CCTs' observations showed $12.5 \%$ non-compliance, whereas none of the observations among the physiotherapist group showed noncompliance with the practices of tracheal suctioning. In conclusion, through an analysis using Fisher Exact tests $\mathrm{x}^{2}$ the P-value (0.607) indicated no significant difference in tracheal suctioning practices among HCPs (Table 2).

\section{Discussion}

In total, $40 \mathrm{HCPs}$ were recruited in the study working in a surgical unit of a TCH in Karachi, Pakistan. The number of nurses was higher in the unit than the CCTs and physiotherapists. Besides this, the findings also highlighted that $70 \%$ of participants were male. This is possible because nursing is no more a female profession. In the modern era, males are also entering this profession. With regard to years of experience, $90 \%$ of the participants had an experience of 1-7 years and only $10 \%$ of the participants fell in the category of more than 10 years of experience. Participants who had experience above ten years were nurses and CCTs who worked as full time employees in the surgical unit. Moreover, internationally studies have been conducted on the same research topic, however; only one study has reported similar years of experience among nurses as the current study. ${ }^{1}$

Each participant was observed twice: one observation done in the morning shift and another was carried out in the evening or night shift. Fifty percent of the observations were carried out in the morning shift, whereas $36 \%$ in the evening, and $14 \%$ of the observations were made in the night shifts.

\section{Compliances with tracheal suctioning protocol}

The current study found that $50 \%$ of the observations fell in the category of partially compliant, whereas only $30 \%$ of the practices showed compliance in the practices, and $20 \%$ of them fell in the category of non-compliance. Besides this, the level of compliance, with regard to phases of suctioning findings, in $62.5 \%$ of the observations, showed non-compliance in the pre-suctioning phase. However, $65 \%$ of them showed compliance in the suctioning phase and $92.5 \%$ of the observation revealed compliance in the post suctioning phase.

\section{Compliance with respect to the individual steps of the tracheal suctioning tool}

Pre-suctioning phase: To assess the need of suctioning it is recommended that thorough assessment of the patient should be done. Assessment is a mandatory action in the pre-suctioning, suctioning, and post suctioning phase. As per the findings of the current study, none of the observations showed compliance in this important aspect of patient care. In fact, $100 \%$ of the observations showed noncompliance in this aspect $(n=40)$. Similarly, a study conducted in southeast England identified that $92.9 \%$ of the participants did not conduct an assessment prior to the suctioning. ${ }^{1}$

Patient preparation, by taking consent or informing the patient prior to the suctioning, ensures smooth execution of the procedures and helps in reducing patients' anxiety. Informing the patient and taking consent prior to the suctioning have been identified as strategies for reducing the anxiety and distress of patients, as they help in gaining maximum outcomes from suctioning. ${ }^{1-4}$ The findings of the study indicated that in only $45 \%$ of the observations there was compliance, whereas $55 \%$ of them showed non-compliance with these practices. However, observations of the morning shift showed higher compliance than the evening/night shifts. Similarly, one study explicated that out of $53.28 \%$ of the nurses from an inensive care unit were unable to communicate and explain procedures to the patients. ${ }^{6}$

Positioning the patient prior to and after the suctioning is important as it facilitates effortless breathing but the findings revealed that in $70 \%$ of the observations there was non-compliance with the practices, this could be due to non-availability of helping staff for the procedure or may be due to shortage of time the staff did not chose to position the patient. However, none of the studies have shared findings with respect to this aspect.

During the process of suctioning it is important to keep the suctioning pressure within recommended guidelines $(100-150 \mathrm{mmHg})$ to prevent mucosal damage; while most of the literature mentions a ranged from $80-150 \mathrm{mmHg} .{ }^{5}$ However, the findings of the study $70 \%$ of the observations indicated non-compliance as they used pressure that was more than $150 \mathrm{mmHg}$. These findings correlate with the study that assessed the competencies of tracheal suctioning practices among nurses, which showed that out of 28 participants 26 used a pressure of $150-200 \mathrm{mmHg} .{ }^{1}$ Another study highlighted that $100 \%$ of the participants used a pressure that was more than $150 \mathrm{mmHg}$ during tracheal suctioning. ${ }^{6}$

Compliance with standard precautions (hand washing and gloving) during, before, and after the tracheal suctioning is vital, as it protects the HCPs and prevents the spread of hospital acquired infections among patients. As per the findings of the current study, $45 \%$ of the observations were non-compliant, whereas practices were more compliant (55\%) in the morning shift as compared to the evening/night shifts, in which $100 \%$ of the observations showed non-compliance. In the researcher's opinion, the reason for non-compliance may be negligence.

Saline instillation is a commonly used intervention before suctioning by the HCPs to liquify the secretion for easy removal. However, none of the studies have yet proved its effectiveness. 
Many studies, though, have been done to assess its adverse effects on patients. The findings of the current study reported that $25 \%$ of the participants still used saline prior to and during the suctioning. This finding is consistent with a study conducted to assess the tracheal suctioning practices of the ICU nurses in Finland, which highlighted that out of 40 nurses who participated in the study, $25 \%$ of them used saline before the suctioning. ${ }^{5}$

Although saline instillation has been identified as a harmful intervention, a survey of 27 different sites in the United States highlighted that $74 \%$ of the centers had a protocol which recommended saline instillation during suctioning. ${ }^{9}$ The findings of a survey indicate that $79 \%$ of the hospitals use saline during suctioning, among which $58 \%$ physiotherapists use saline in their practices, while saline use by nurses and other medical staff was $42 \% .{ }^{8}$ In the current study, an analysis of each step has not been done according to the category of HCPs; however, the use of saline varies among HCPs.

Hyperoxygenation is a very important intervention during the whole process of suctioning as it prevents hypoxemia caused by suctioning in patients. The findings of the current study showed that in $70 \%$ of the observations there was non-compliance before and in $60 \%$ after the suctioning. However, a study conducted in Finland showed that $57.5 \%$ of the participants hyperoxygenate patients prior to the suctioning, whereas, $62.5 \%$ in post suctioning phase do so which is somewhat consistent with the findings of the current study. ${ }^{5}$ Moreover, a study done in a hospital in south east England highlighted that out of 28 nurses 10 were knowledgeable regarding hyperoxygenation, whereas, only two nurses were observed doing so in the practice. ${ }^{1}$ On the other hand, a study done in Ireland showed that the majority of the nurses from the cardiac intensive care unit (94\%) and general intesive care GICU (79\%) were compliant with this step of tracheal suctioning. ${ }^{6}$

Suctioning phase: Hospital associated infections are the most common complication of hospitalization, therefore, it is important to follow the aseptic technique during suctioning to prevent the spread of nosocomial infections. In the present study, $60 \%$ of the observationsshowed that the participants failed to maintain the sterility of the catheter before insertion into the airway. The findings correlate with the study conducted in Ireland, which reported that $59 \%$ of the CICU and $29 \%$ of GICU nurses failed to maintained catheter sterility before insertion (Kelleher \& Andrews. 2006). However, a study conducted in Finland reported that $61.5 \%$ of the nurses showed compliance with this step of suctioning phase. ${ }^{5}$

The suction catheter should not be inserted deeply into the trachea as it causes cough and vagal stimulation at the bifurcation of the trachea (Crina) and if resistance is felt the catheter should be pulled out $1 \mathrm{~cm}$ before applying the suction..$^{1,4,10}$ However, $45 \%$ of the observations showed non-compliance and $55 \%$ of them indicated compliance. None of the studies have assessed this critical step of tracheal suctioning, which can lead to a life threatening situation.

It is important to note that the suction vacuum should always be applied during catheter withdrawal, as vacuum during the insertion of the catheter can damage the mucosal wall of the trachea. In the present study $52.5 \%$ of the observations showed compliance. However, the study conducted in Finland reported that $100 \%$ of the GICU and CICU nurses were compliant in this step. ${ }^{6}$ Whereas, Day et al. ${ }^{1}$ reported that out of 28 nurses 18 applied suction pressure while withdrawing the catheter.

Post-suctioning phase: In order to evaluate the effectiveness of tracheal suctioning, assessment is a critical action that HCPs should perform following the suction procedure. As per the findings of the current study, $90 \%$ of the observations indicated non-compliance. Similar findings have been reported by Day et al. ${ }^{1}$ that out of 28 nurses 26 were knowledgeable about assessment in the post suctioning phase, however, only one nurse being observed as performing the assessment. Moreover, Jansson et al. ${ }^{5}$ have reported that none of the participants carried out the assessment (100\%), and Kelleher and Andrews ${ }^{6}$ share that $94 \%$ of CICU and $93 \%$ of GICU nurses failed to assess the patient in the post suctioning phase.

Documentation is an important aspect after the intervention. It facilitates reporting and maintaining continuity of patient care. As per the the findings of the current study, $92.5 \%$ of the observations revealed non-compliances however, only $7.5 \%$ of them showed compliance in the morning shift. However, none of the studies have shared findings regarding documentation.

\section{Difference of compliance among HCPs}

The present study identified that $58.3 \%$ of the nurses, $16.7 \%$ of the CCTs, and $25 \%$ of the physiotherapists were compliant with the overall tracheal suctioning practices. Whereas, $87.5 \%$ of the nurses and $12.5 \%$ of the CCts showed non-compliance with the practices. However, none of the observations from the physiotherapist group fall in the category of non-compliance. However, P value of 0.607 does not show a significant difference in the overall practices of tracheal suctioning.

\section{Conclusion}

In conclusion, this study is the first of its kind in Pakistan in which the tracheal suctioning practices of HCPs, which included CCTs and physiotherapists, besides nurses working at a surgical care unit of a TCH were assessed. Differences in the level of compliance with tracheal suctioning guidlines, with regard to the professional characteristics of the study participants and the working shifts, were probed. This study has contributed in the development of evidence based literature on this topic in the national context, and this study could be viewed as another significant step towards fulfilling the mandate of patient safety.

\section{Funding}

None.

\section{Acknowledgments}

None.

\section{Conflicts of interest}

The author declares that there is no conflict of interest.

\section{References}

1. Day T, Farnell S, Wilson-Barnett J. Suctioning: a review of current literature recommondation. Intensive and Critical Care Nursing. 2002;18(2):79-89.

2. Day T, Farnell S, Haynes S, et al. Tracheal suctioning: an exploration of nurses' knowledgeand competence in acute and high dependency ward areas. J Adv Nurs. 2002;39(1):35-45.

3. Day T, Wainwright SP, Wilson-Barnett J, et al. An evaluation of teaching Intervention to improve the practice of endotracheal suctioning in intensive care unit. J Clin Nurs. 2001;10;682-696. 
4. Freeman S. Care of adult patients with a temporary trachesotomy. Nursing Standard. 2011;26(2):49-56.

5. Jansson M,Ala-Kokko T, Ylipalosaari P, et al. Evaluation of endotrachealsuctioning practices of critical-care nurses - An observational correlation study. Journal of Nursing Education and Practice. 2013;3(7):99-105.

6. Kelleher S, Andrews T. An observational study on the open-system endotracheal suctioning practices of critical care nurses. J Clin Nurs. 2008;17(3):360-369.

7. Polit DF, Beck CT. Nursing research: Generating and assessing evidence for nursing practice. Philadelphia: Lippincott Williams \& Wilkins; 2008.
8. Reeve JC, Davies N, Freeman J, et al. The use of normal saline instillation in the intensive care unit by physiotherapists: a survey of practice in New Zealand. Journal of Physiotherapy. 2007;35(3):119-125.

9. Sole ML, Byers JF, Zhang Y, Banta, et al. A Multisite Survey of Suctioning Techniques and Airway Management Practices. American Journal of Critical Care. 2003;12(3):220-230.

10. Wood CJ. Endotracheal suctioning: a literature review. Journal of Intensive and Critical Care Nursing. 1998;14(3):124-136. 